\title{
In vivo melanoma depth detection by a handheld photoacoustic microscope
}

Yong Zhou, Wenxin Xing, Konstantin I. Maslov, Lynn A. Cornelius, Lihong V. Wang

Yong Zhou, Wenxin Xing, Konstantin I. Maslov, Lynn A. Cornelius, Lihong V. Wang, "In vivo melanoma depth detection by a handheld photoacoustic microscope," Proc. SPIE 9323, Photons Plus Ultrasound: Imaging and Sensing 2015, 932304 (11 March 2015); doi: 10.1117/12.2076684

SPIE. Event: SPIE BiOS, 2015, San Francisco, California, United States 


\title{
In Vivo Melanoma Depth Detection by a Handheld Photoacoustic Microscope
}

Yong Zhou, ${ }^{\mathrm{a}}$ Wenxin Xing, ${ }^{\mathrm{a}}$ Konstantin I. Maslov, ${ }^{\mathrm{a}}$ Lynn A. Cornelius, ${ }^{\mathrm{b} *}$ and Lihong V. Wang ${ }^{\mathrm{a}}$

${ }^{a}$ Washington University in St. Louis, Department of Biomedical Engineering, Optical Imaging

Laboratory, 1 Brookings Drive, Campus Box 1097, St. Louis, Missouri 63130

${ }^{b}$ Washington University School of Medicine, Division of Dermatology, 660 S. Euclid, Campus

Box 8123, St. Louis, Missouri 63110

*Correspondence: Lynn A. Cornelius (melanoma studies), LCORNELI@DOM.wustl.edu;

Lihong V. Wang (photoacoustic imaging), lhwang@wustl.edu

\begin{abstract}
We developed a handheld photoacoustic microscope (PAM) to detect melanoma and determine tumor depth in nude mice in vivo. Compared to our previous PAM system for melanoma imaging, a new light delivery mechanism is introduced to improve light penetration. We show that melanomas with $4.1 \mathrm{~mm}$ and $3.3 \mathrm{~mm}$ thicknesses can be successfully detected in phantom and in vivo experiments, respectively. With its deep melanoma imaging ability and novel handheld design, this system is promising for clinical melanoma diagnosis, prognosis, and surgical planning for patients at the bedside.
\end{abstract}

Keywords: Handheld, photoacoustic microscope, melanoma, depth

\section{Introduction}

Melanoma is now the fifth most common cancer in the United States [1,2], where there were about 76,690 men and women diagnosed with melanoma in 2013 alone [2,3]. Although melanoma accounts for less than 5\% of all skin cancers [2], it causes more than $75 \%$ of deaths related to skin cancer [1]. In addition, the incidence of melanoma is increasing faster than that of any other cancer [4]. As a result, there is an increased demand for accurate diagnosis, prognosis, and treatment of melanoma.

The thickness of a melanoma is a critical parameter for determining definitive treatment and diagnosis [5]. Based on its thickness, the tumor (T) classification in the tumor-node-metastasis (TNM) staging system can be further divided into T1 $(\leq 1.0 \mathrm{~mm})$, T2 $(1.01-2.0 \mathrm{~mm}), \mathrm{T} 3(2.01-4.0 \mathrm{~mm})$, and T4 $(>4.0 \mathrm{~mm})$ [2]. As the thickness increases, so do the chances for developing metastatic disease, and thus, disease mortality. Current diagnoses are dependent upon clinical suspicion, subsequent biopsy, and histologic examination. It has been shown that clinical examination alone may miss certain tumors, and conversely, many benign lesions may be unnecessarily biopsied $[6,7]$. In trained hands, the use of handheld dermoscopy in the clinic has aided in the diagnosis of melanoma by facilitating the evaluation of surface characteristics of pigmented lesions [8]. It does not, however, enable the determination of tumor thickness and vertical growth of the melanoma, which are important tumor characteristics when it comes to determining surgical treatment. In fact, the surgeon is often met with the confounding situation where a pigmented lesion may be partially biopsied and the diagnosis of melanoma is made. Consequently, the true tumor depth may not be determined due to sampling only part of the tumor, leading to an inaccurate measurement. Thus, a wide local excision (WLE) based upon a provisional tumor depth may not be appropriate, and a second surgery may be required to adequately treat the tumor. Thus, a precise in vivo measurement of tumor depth, prior to definitive surgery, would facilitate appropriate patient treatment and help avoid further surgeries.

In the past few years, several noninvasive imaging techniques have been developed for melanoma diagnoses [5, 6 , 9]. However, all of them have limitations. Optical methods, such as dermoscopy [8], total-body photography (TBP) [10], optical coherence tomography [11], scanning confocal microscopy [12], and two-photon microscopy [13], do

Photons Plus Ultrasound: Imaging and Sensing 2015, edited by Alexander A. Oraevsky, Lihong V. Wang

Proc. of SPIE Vol. 9323, 932304 - @ 2015 SPIE · CCC code: 1605-7422/15/\$18 doi: $10.1117 / 12.2076684$

Proc. of SPIE Vol. 9323 932304-1 
not have sufficient penetration to determine melanoma depth because they can image only in the ballistic regime. High frequency ultrasound can provide deeper penetration than optical methods [14]. However, because there is often no sufficient difference in acoustic impedance between melanoma and normal tissues, the image contrast for melanoma is poor. Magnetic resonance imaging (MRI) and positron emission tomography (PET) have also been used in melanoma diagnoses $[15,16]$. However, they are both expensive and have poor resolution in the skin. Meanwhile, PET has to use tracers for sufficient contrast and can only detect tumors as large as $\sim 1 \mathrm{~cm}$ in dimension, which encompasses only a tiny set of $\mathrm{T} 4$ primary melanomas and misses all $\mathrm{T} 1-\mathrm{T} 3$ ones.

Recently, photoacoustic microscopy (PAM) has shown strong ability in detecting skin vasculatures with high contrast and deep penetration [17-22]. In PAM, short laser pulses illuminate the object. Following the absorption of photons, ultrasonic waves are induced thermoelastically through the photoacoustic (PA) effect. Because melanin in most melanomas has a very broad and strong absorption spectrum, it can be imaged by PAM with high contrast. Meanwhile, because acoustic scattering is low in the tissue, high resolution can be achieved with deep penetration.
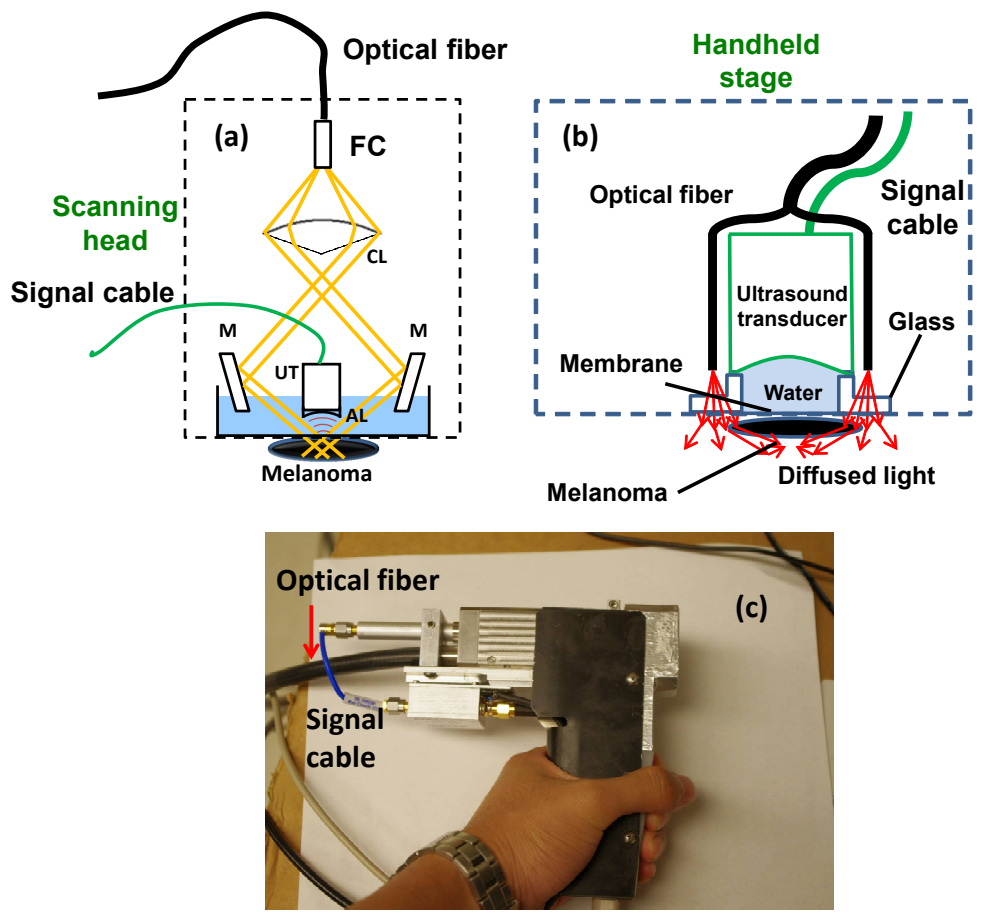

Fig. 1. (a) Previous photoacoustic microscopy (PAM) system for melanoma detection. (b) Components held by the translation stage in the handheld probe. (c) A photograph of the handheld probe.

Previously, we reported that cutaneous pigmented lesions, melanoma, and the surrounding vasculature could be successfully detected by using dual-wavelength PAM [23]. In that study, light was transmitted through the whole melanoma, resulting in a high attenuation, with only very few photons reaching the base of the melanoma (true tumor depth), as shown in Fig. 1(a). In the end, PA signals were very strong from the surface of the tumor but very weak from the deepest portion. The experimental results showed that a melanoma phantom with a maximum thickness of $1.27 \mathrm{~mm}$ could be imaged, which covered only the T1 classification. In this report, we describe a new PAM system with more efficient light illumination [24]. By allowing light to bypass the center of the tumor, more photons reach the inferior depth of the melanoma. Equally important, this system is designed to be handheld, facilitating clinical usage.

\section{System}

Fig. 1(b) shows a schematic of the handheld photoacoustic probe. Compared to our previous PAM system, the light is delivered into the tissue normally instead of obliquely. Thus, the light propagates around the melanoma instead of going through it. Since the surrounding tissue has high optical scattering but low absorption, light reaching the 
bottom boundary of the melanoma is increased. The incident beam has an annular shape with $8 \mathrm{~mm}$ inner and 20 $\mathrm{mm}$ outer diameters. The transducer, with $25 \mathrm{MHz}$ central frequency and $100 \%$ nominal bandwidth, detects the PA signals. The focal length and diameter of the detector are $12.7 \mathrm{~mm}$ and $6.4 \mathrm{~mm}$, respectively, yielding a numerical aperture (NA) of 0.25 . Both the optical fibers and transducer are fixed on a motorized translation stage. Each laser pulse generates a one-dimensional depth-resolved image (A-line). A two-dimensional cross sectional image is acquired by linear scanning of the region of interest. As shown in Fig. 1(b), the motor, translation stage, ultrasonic transducer, and optical fibers are all incorporated in a handheld probe for easy operation. A photograph of the handheld probe is shown in Fig. 1(c).

(a)
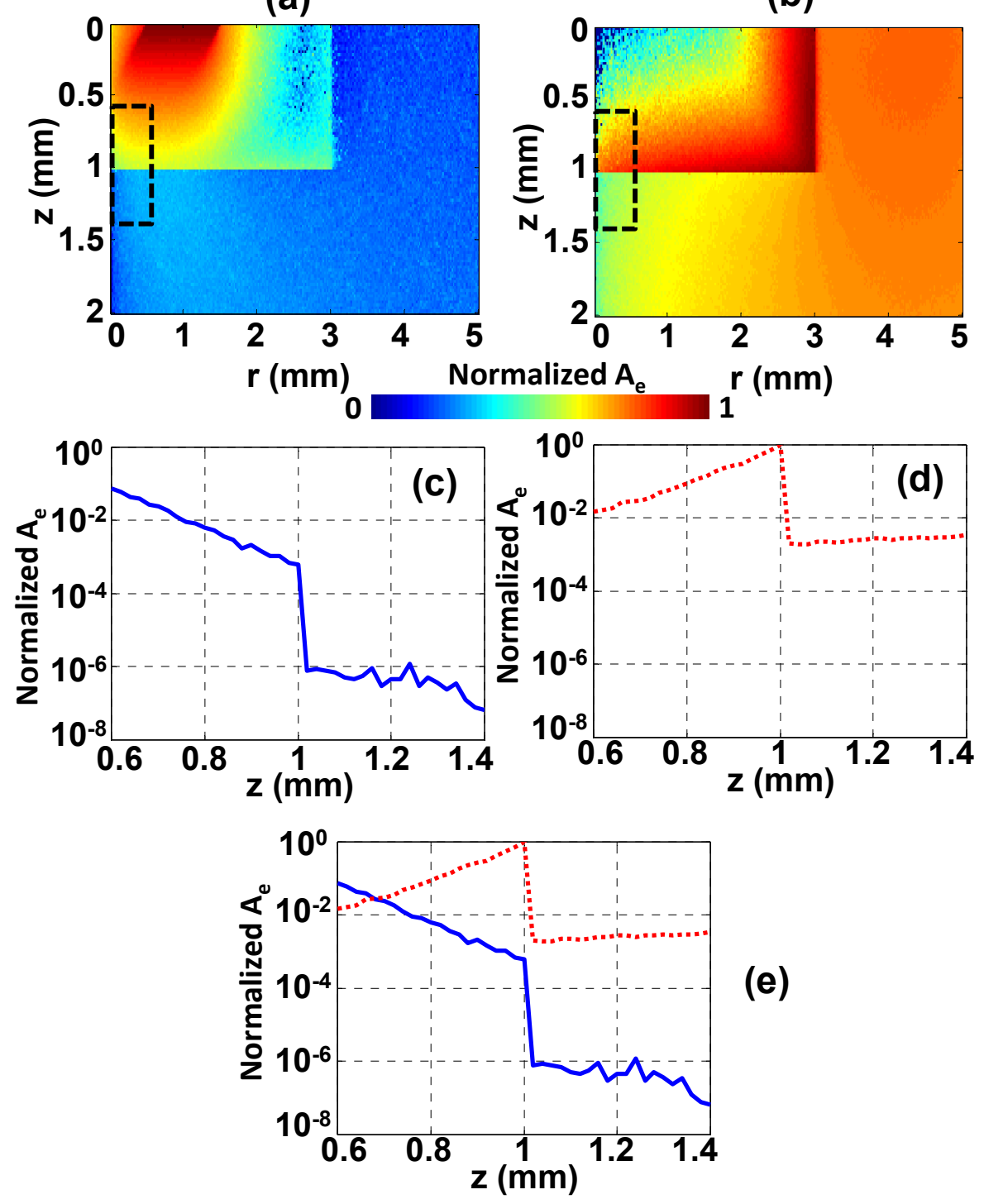

(e)

Fig. 2. Monte Carlo simulations of the old and new PAM systems for melanoma imaging. Specific heat absorption $\left(\mathrm{A}_{\mathrm{e}}\right)$ distributions in the old (a) and new (b) PAM systems with the same incident energy. (c-d) Averaged $A_{e}$ in the blackdashed-square areas (shown in (a-b)) at the bottom boundary of the melanoma phantoms showing that the melanoma is much more strongly illuminated at the bottom boundary using the new PAM system. (e) Combined curves in (c) and (d). 

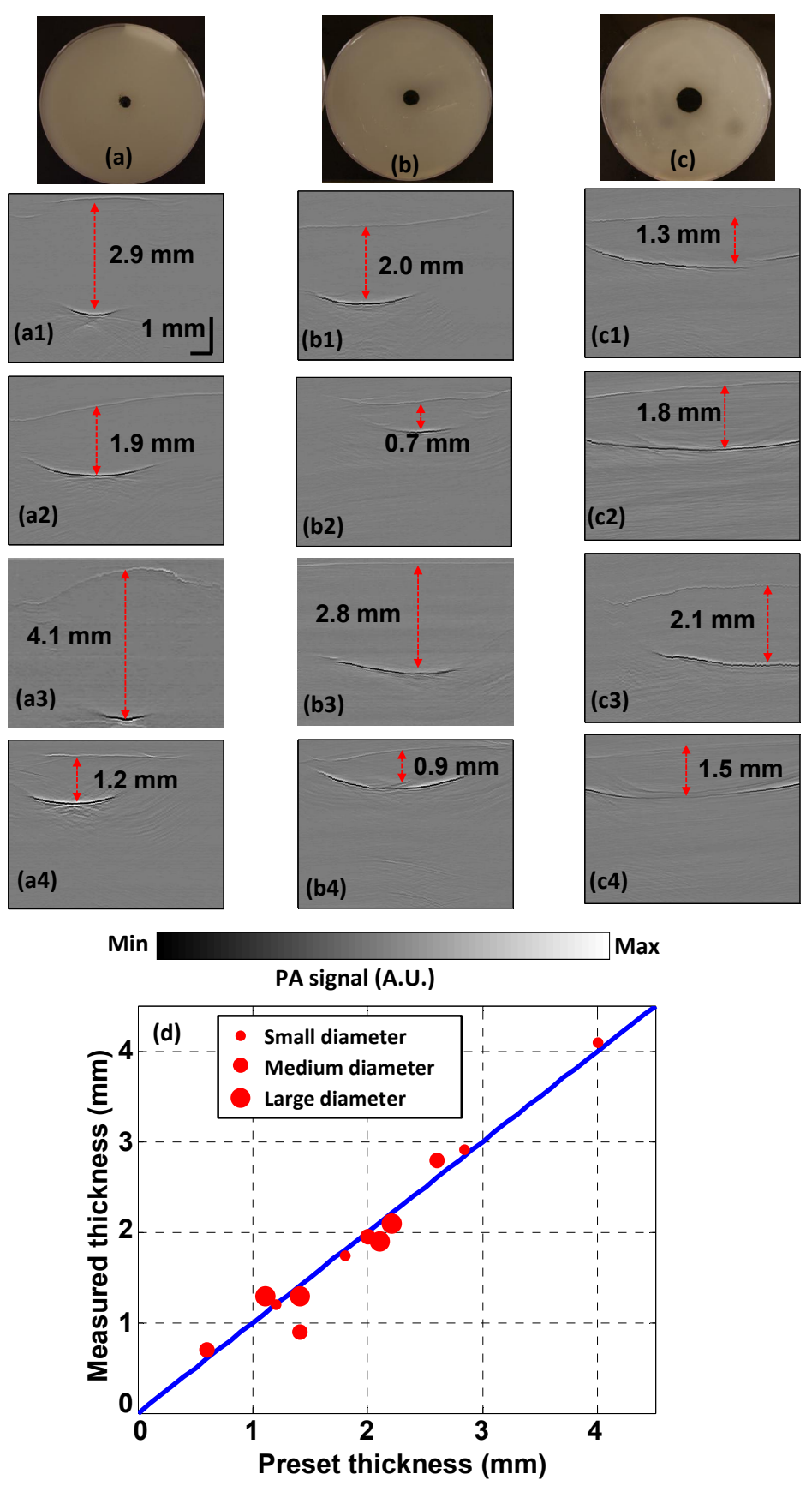

Fig. 3. Handheld PAM of melanoma phantoms. Photos of melanoma phantoms with different diameters ((a), $7 \mathrm{~mm}$; (b), $9.5 \mathrm{~mm}$; and (c), $14 \mathrm{~mm}$ ). (a1-a4), (b1-b4), and (c1-c4): PAM images of different thicknesses of melanoma phantoms with the same diameter as in (a), (b), and (c), respectively. (d) Measured thicknesses of melanoma phantoms versus preset thicknesses.

\section{Simulation}

We use Monte Carlo simulations to show the advantage of the new light illumination mechanism [25]. In the old light illumination mechanism (hereinafter referred to as case 1), the incident angle was set to $45^{\circ}$, which was typical in the previous PAM system. In the new light illumination mechanism (hereinafter referred to as case 2), the incident angle was set to $90^{\circ}$. In both simulations, the same incident energies were used. The same melanoma phantom, with $1 \mathrm{~mm}$ thickness and $6 \mathrm{~mm}$ diameter, was used in both simulations. The simulation parameters 
(absorption coefficient of the melanoma phantom, $70 \mathrm{~cm}^{-1}$; absorption coefficient of the background, $0.1 \mathrm{~cm}^{-1}$; scattering coefficients of the melanoma phantom and background, $100 \mathrm{~cm}^{-1}$; scattering anisotropy of the melanoma phantom and background, 0.9) were selected based on normal tissue optical properties at $650 \mathrm{~nm}$ wavelength [26, 27]. As shown in Fig. 2(a), in case 1, the specific optical absorption $\left(A_{e}\right)$ decreases sharply in the melanoma phantom in the depth direction. However, as shown in Fig. 2(b), in case 2, Ae actually increases in the melanoma phantom in the depth direction. A detailed examination of the melanoma at its bottom boundary is shown in Fig. 2(c-e). As shown in Fig. 2(a) and (b), the same black-dashed-square area was chosen to calculate the average $A_{e}$ for comparison between case 1 and case 2 . As shown in Fig. (e), the average $A_{e}$ at the bottom boundary of the melanoma in case 1 was 1660 times weaker than that in case 2. In both thermal and stress confinements, the PA signal is proportional to $A_{e}$. Thus, the PA signal in case 2 is 1660 times stronger than that in case 1 . Because the light delivery is the only difference between the systems, their noise levels should be the same. Thus, the corresponding signal-to-noise ratio of the melanoma at the bottom boundary in the black-dashed-square area in case 2 is 1660 times greater than that in case 1 . The results show that, with the new illumination mechanism, the bottom boundary of the melanoma can be much more sensitively detected.
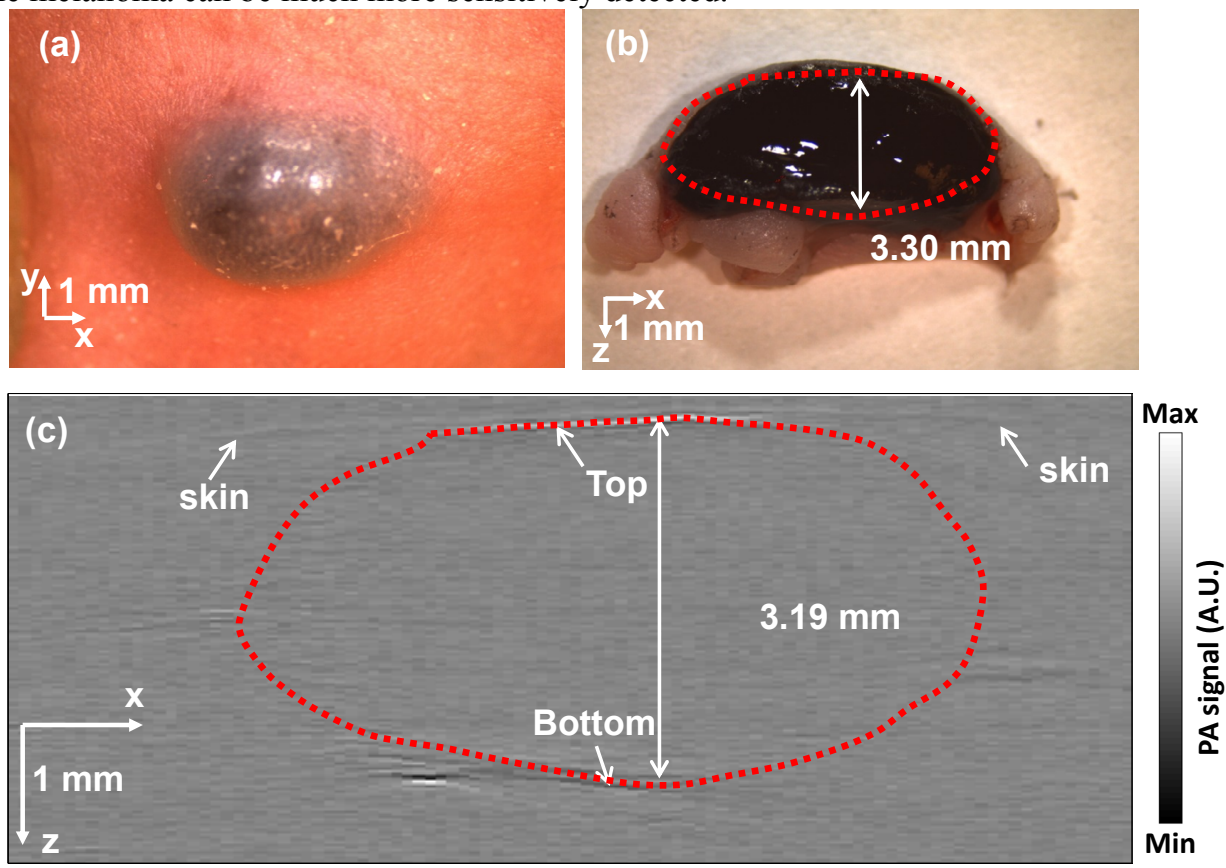

Fig. 4. Handheld PAM of melanoma in a nude mouse in vivo. (c) Photograph of the melanoma taken in vivo. (b) Photograph of the excised melanoma. The red dots outline the melanoma. (c) PAM image of the melanoma clearly showing both the top and bottom boundaries. The red dots outline the melanoma according to the shape of the excised melanoma in its photograph (b).

\section{Phantom experiment}

Phantom experiments were conducted to demonstrate the ability of the handheld PAM system to measure deep melanomas. Three different diameters $(7 \mathrm{~mm}, 9.5 \mathrm{~mm}$, and $14 \mathrm{~mm})$ of melanoma phantoms were prepared with varied thicknesses, shown in Fig. 4(a)-(c). All the phantoms were made from a black ink and gelatin mixture, which had an absorption coefficient of $70 \mathrm{~cm}^{-1}$ at $650 \mathrm{~nm}$, close to the real melanoma absorption coefficient. The background was made of a gelatin and intralipid mixture with a high scattering coefficient to mimic real tissue. As shown in Fig. 4(a)-(c), our handheld system successfully detected both the top and bottom boundaries of all the melanoma phantoms. The maximum and minimum detected thicknesses of the phantoms were $0.7 \mathrm{~mm}$ and $4.1 \mathrm{~mm}$, respectively. Thus, melanoma phantoms with all the T-classifications in the staging system have been measured. Fig. 4(d) shows that our measured thicknesses agreed well with the preset values. Thus, the handheld PAM system can image deeply seated melanoma phantoms with high accuracy. 


\section{In vivo experiment}

We also imaged mice with melanoma to show the in vivo detection ability of the handheld PAM system. Here we focused only on imaging deep melanoma to show the advantage of the new PAM system over our previous system. Melanoma B16 cells were subcutaneously injected into a nude mouse on the dorsal side (Hsd:Athymic NudeFoxn1NU, Harlan Co.; body weight: $20 \mathrm{~g}$ ) to grow melanoma in the skin. All experimental animal procedures were carried out in conformity with the laboratory animal protocol approved by the School of Medicine Animal Studies Committee of Washington University in St. Louis. During image acquisition, the animal, warmed by an infrared lamp, was kept motionless using a breathing anesthesia system (E-Z Anesthesia, Euthanex). Fig. 4(a) shows the melanoma in vivo. As shown in Fig. 4(b), the real depth of the melanoma was measured to be $3.30 \mathrm{~mm}$ after excision. Fig. 4(c) shows that both the top and bottom boundaries of the melanoma and the skin surface can be clearly seen in the PA image. The measured depth was $3.19 \mathrm{~mm}$, which is very close to the real depth. Therefore, the handheld PAM system can accurately detect the depth of deeply seated melanoma in vivo.

\section{Discussion and Conclusion}

Compared to our previous PAM system for melanoma imaging, the new system can detect the depth of thicker melanomas by bypassing light around the tumor. In addition, a handheld design makes our new PAM system more suitable for clinical application. As shown in the phantom experiment, melanoma as thick as $4.1 \mathrm{~mm}$ can be detected, and in the in vivo experiment, melanoma with $3.30 \mathrm{~mm}$ thickness was imaged. Our measurements also show very high accuracy. As important, the improvement of the handheld system includes increasing the imaging speed. The current system employs a $10 \mathrm{~Hz}$ laser, which limits the imaging speed. For clinical use, a rapid imaging system is always preferred, which can be achieved by using a pump laser with high pulse repetition rate (e.g., $1 \mathrm{kHz}$ ). We propose that the use of handheld PAM imaging, to aid in the determination of tumor depth, will facilitate the surgical treatment management of melanoma.

\section{Acknowledgments}

The authors would like to thank Prof. James Ballard for manuscript editing. This work was sponsored in part by National Institutes of Health grants DP1 EB016986 (NIH Director's Pioneer Award), R01 CA186567 (NIH Director's Transformative Research Award), R01 EB016963, and R01 CA159959. L.W. has a financial interest in Microphotoacoustics, Inc. and Endra, Inc., which, however, did not support this work. K.M. has a financial interest in Microphotoacoustics, Inc.

\section{References}

1. $\quad$ A. F. Jerant, J. T. Johnson, C. D. Sheridan and T. J. Caffrey, "Early detection and treatment of skin cancer," American family physician 62(2), 357-368, 375-356, 381-352 (2000).

2. E. M. Dunki-Jacobs, G. G. Callendar and K. M. McMasters, "Current management of melanoma," Curr. Prob. Surg. 50(8), 351-382 (2013).

3. American Cancer Society, http://www.cancer.org/.

4. K. M. Rubin, "Melanoma Staging: A Review of the Revised American Joint Committee on Cancer Guidelines," Journal of the Dermatology Nurses' Association 2(6), 6 (2010).

5. L. Smith and S. MacNeil, "State of the art in non-invasive imaging of cutaneous melanoma," Skin Res Technol 17(3), 257-269 (2011).

6. $\quad$ P. Guitera and S. W. Menzies, "State of the art of diagnostic technology for early-stage melanoma," Expert Rev Anticanc 11(5), 715-723 (2011).

7. I. H. Wolf, J. Smolle, H. P. Soyer and H. Kerl, "Sensitivity in the clinical diagnosis of malignant melanoma," Melanoma Res 8(5), 425-429 (1998).

8. C. Benvenuto-Andrade, S. W. Dusza, A. L. C. Agero, A. Scope, M. Rajadhyaksha, A. C. Halpern and A. A. Marghoob, "Differences between polarized light dermoscopy and immersion contact dermoscopy for the evaluation of skin lesions," Arch Dermatol 143(3), 329-338 (2007).

9. M. A. Calin, S. V. Parasca, R. Savastru, M. R. Calin and S. Dontu, "Optical techniques for the noninvasive diagnosis of skin cancer," J Cancer Res Clin 139(7), 1083-1104 (2013).

10. A. C. Halpern, "Total body skin imaging as an aid to melanoma detection," Semin Cutan Med Surg 22(1), 2-8 (2003). 
11. T. Gambichler, P. Regeniter, F. G. Bechara, A. Orlikov, R. Vasa, G. Moussa, M. Stucker, P. Altmeyer and K. Hoffmann, "Characterization of benign and malignant melanocytic skin lesions using optical coherence tomography in vivo," J Am Acad Dermatol 57(4), 629-637 (2007).

12. S. Segura, S. Puig, C. Carrera, J. Palou and J. Malvehy, "Development of a two-step method for the diagnosis of melanoma by reflectance confocal microscopy," J Am Acad Dermatol 61(2), 216-229 (2009).

13. E. Dimitrow, M. Ziemer, M. J. Koehler, J. Norgauer, K. Konig, P. Elsner and M. Kaatz, "Sensitivity and Specificity of Multiphoton Laser Tomography for In Vivo and Ex Vivo Diagnosis of Malignant Melanoma," J Invest Dermatol 129(7), 1752-1758 (2009).

14. W. Dummer, H. J. Blaheta, B. C. Bastian, T. Schenk, E. B. Brocher and W. Remy, "Preoperative Characterization of Pigmented Skin-Lesions by Epiluminescence Microscopy and High-Frequency Ultrasound," Arch Dermatol 131(3), 279-285 (1995).

15. J. Bittoun, B. Querleux and L. Darrasse, "Advances in MR imaging of the skin," Nmr Biomed 19(7), 723730 (2006).

16. T. Z. Belhocine, A. M. Scott, E. Even-Sapir, J. L. Urbain and R. Essner, "Role of nuclear medicine in the management of cutaneous malignant melanoma," J Nucl Med 47(6), 957-967 (2006).

17. Y. Zhou, J. J. Yao and L. H. V. Wang, "Optical clearing-aided photoacoustic microscopy with enhanced resolution and imaging depth," Opt. Lett. 38(14), 2592-2595 (2013).

18. L. H. V. Wang and S. Hu, "Photoacoustic Tomography: In Vivo Imaging from Organelles to Organs," Science 335(6075), 1458-1462 (2012).

19. Y. Zhou, C. Zhang, D. K. Yao and L. H. V. Wang, "Photoacoustic microscopy of bilirubin in tissue phantoms," J. Biomed. Opt. 17(12), (2012).

20. Y. Zhou, J. Yao, K. I. Maslov and L. V. Wang, "Calibration-free absolute quantification of particle concentration by statistical analyses of photoacoustic signals in vivo," J Biomed Opt 19(3), 37001 (2014).

21. Y. Zhou, X. Yi, W. Xing, S. Hu, K. Maslov, and L. V. Wang, "Microcirculatory changes identified by photoacoustic microscopy in patients with complex regional pain syndrome type I after stellate ganglion blocks," J. Biomed. Opt. 19(8), 086017 (2014).

22. Y. Zhou, J. Liang, K. I. Maslov, and L. V. Wang, "Calibration-free in vivo transverse blood flowmetry based on cross correlation of slow time profiles from photoacoustic microscopy," Opt. Lett. 38(19), 3882-3885 (2013).

23. J. T. Oh, M. L. Li, H. F. Zhang, K. Maslov, G. Stoica and L. H. V. Wang, "Three-dimensional imaging of skin melanoma in vivo by dual-wavelength photoacoustic microscopy," J. Biomed. Opt. 11(3), (2006).

24. Y. Zhou, W. Xing, K. I. Maslov, Lynn A. Cornelius, and L. V. Wang, "Handheld photoacoustic microscopy to detect melanoma depth in vivo," Opt. Lett. 39(16), 4731-4734 (2014).

25. L. H. Wang, S. L. Jacques and L. Q. Zheng, "CONV - convolution for responses to a finite diameter photon beam incident on multi-layered tissues," Comput Meth Prog Bio 54(3), 141-150 (1997).

26. S. L. Jacques and D. J. Mcauliffe, "The Melanosome - Threshold Temperature for Explosive Vaporization and Internal Absorption-Coefficient during Pulsed Laser Irradiation," Photochem Photobiol 53(6), 769-775 (1991).

27. L. V. Wang and H. Wu, Biomedical Optics: Principles and Imaging, WILEY (2007). 